Voix et Images

volxetimages

\title{
Montréal, le samedi 2 mai 1942 : La grosse femme d'à côté est enceinte, de Michel Tremblay
}

\section{Jacques Allard}

Volume 4, numéro 3, avril 1979

Louis-Philippe Hébert

URI : https://id.erudit.org/iderudit/200176ar

DOI : https://doi.org/10.7202/200176ar

Aller au sommaire du numéro

Éditeur(s)

Les Presses de l'Université du Québec

ISSN

0318-9201 (imprimé)

1705-933X (numérique)

Découvrir la revue

Citer ce compte rendu

Allard, J. (1979). Compte rendu de [Montréal, le samedi 2 mai 1942 : La grosse femme d'à côté est enceinte, de Michel Tremblay]. Voix et Images, 4(3), 537-540. https://doi.org/10.7202/200176ar d'utilisation que vous pouvez consulter en ligne.

https://apropos.erudit.org/fr/usagers/politique-dutilisation/ 


\section{Montréal, le samedi 2 mai 1942 : \\ La grosse femme d'à côté est enceinte, de Michel Tremblay}

La grosse femme d'à côté est enceinte, dit le dernier roman de $M$. Tremblay. La couverture du livre le répète qui nous montre une femme fatiguée, dans la quarantaine avancée, cheveux relevés en chignon, et dont les rondeurs bien accusées par la robe de chambre, qui descend jusqu'à ses pantoufles, sont calées dans un gros fauteuil anglais. Près d'elle, une grande fenêtre au store levé découvre une autre fenêtre, celle d'une maison voisine, bordée d'un balcon et, plus loin, d'autres maisons encore percées de fenêtres. La grosse femme a le regard tourné vers l'intérieur et ne semble pas près de se lever. "Eppur si muove", dit Galilée mis en épigraphe, comme pour sourire ou nous prévenir que tout va pourtant bouger, même la grosse femme sans nom.

Et le récit commence avec une autre inscription préalable: 2 mai 1942. Mais it n'y en aura plus d'autre, pas même d'indications de chapitres : tout viendra en soixante-six petits épisodes ou moments d'un beau samedi, séparés par des espaces blancs. Nous sommes rue Fabre, entre la rue Gilford et la rue Mont-Royal, dans l'est de Montréal, au cœur du royaume de Tremblay.

C'est le temps de la conscription et des femmes enceintes, l'année même de la naissance de l'auteur. Le temps des femmes et des femmelettes (hommes qui ont fécondé les premières pour éviter l'enrôlement, grands enfants encore attachés à leur mère ou à leur sœur), mais aussi celui de la tendre enfance et du début de l'adolescence. C'est le printemps. Et il est même précoce.

Se déroule ainsi, au fil d'un jour, les petites misères et pareilles joies du menu peuple montréalais. Le texte énumère, les uns après les autres, les événements propres à chacun ou à chaque ménage de la rue Fabre et des alentours. La langue parle, comme prévu, colorée, pittoresque à souhait: c'est la parole même du quartier, prise sur le vif ou encore redonnée par l'indirect libre. Le narrateur déploie les petits drames, les entremêlent, trouve toujours la bonne scène à faire, les mots vrais (presque toujours), sans qu'il y ait de grand suspense mais. sans que l'intérêt ne diminue. II entraîne son lecteur dans la promenade qui conduit les enfants et plusieurs adultes jusqu'au parc Lafontaine. Ou alors, pour faire contraste, il nous 
confine de temps à autre dans les maisons closes, surtout celles des maternités pudiques, honteuses ou même maladives. Et la fresque culminera dans le retour des promeneurs, dans leur rassemblement pour le souper chez Victoire ainsi que dans la sortie des femmes enceintes, réunies sur le balcon de la même maison, à l'invitation de la grosse femme qui y habite. La maison de la tribu de Victoire retrouve alors le rôle central qu'elle avait dès le début, à l'instar de ses habitants (trois générations s'y entassent) dont les mouvements traversent tout le récit, regroupent tous les fils narratifs.

Comme on le voit, ce récit naturaliste est bien ficelé. Mais il faut pourtant se méfier de la «naturalité » de ce beau et bon discours populaire. Les réflexions prêtées au chat Duplessis et à son ennemi Godbout [...] pourraient paraître fantaisistes, divertissantes ou même "fantastiques». Prêtons plus d'attention aux tricoteuses, Rose, Violette, Mauve, et à leur mère Florence. Ces vieilles commères ouvrent et ferment le récit. Au début, elles semblent être de bonnes fées, sorte de grand-mères d'emprunt qui veillent au bien-être des bébés à venir : les filles tricotent des «pattes» de laine (chaussons) et la mère contrôle la qualité du tricot.

On se rend toutefois bientôt compte du caractère particulier de ces super-grand-mères qui, en connaissant tout du passé, du présent et même de l'avenir de leurs voisins, restent inconnues de tout le monde sauf du chat Duplessis, du jeune enfant Marcel, de Victoire (qui croit voir l'une d'elles, en une sorte d'hallucination) et du narrateur, bien entendu (qui les contredira à une reprise). Ce groupe constitue sans aucun doute une représentation des Moires, des Parques, des poètes antiques et fait concurrence à l'instance narrative elle-même: le narrateur omniscient doit tenir compte de l'action de ces Immortelles sans pouvoir par ailleurs "diriger" leur comportement. Bien sûr, pour le narrataire, tout cela est «fiction". Mais l'analyste trouvera profit à les suivre: leur tricot est celui-là même du récit, puisque leurs principales interventions (lexies $1,13,23,45,66$ ) invitent à un découpage narratif en quatre parties (deux brèves, deux longues) qui marquent le déroulement du temps et des épisodes: le matin, le midi, l'après-midi et la soirée. Autres exemples de leur rôle “directif ": Violette, la tricoteuse maladroite qui referme la "patte", en en faisant une boule de laine, "tricote" la mort de la vieille prostituée Ti-Lou, comme le signalent sa mère et le narrateur qui reprend la parole (p. 283); plus tôt, au début de la troisième partie, la même Violette, troublée par ses souvenirs, a quitté son tricot et le balcon, laissant sa balle de laine sur le perron et permettant à Duplessis de la "massacrer", la "déchirer", la «mordre": or, c'est précisément dans cette partie qu'on nous fera le récit des souffrances de beaucoup de personnages: les troubles de la puberté chez Richard, le mal d'amour chez Albertine, les douleurs de Ti-Lou l'amputée (à force d'avoir les jambes en l'air, elle en a perdu une!), I'humiliation de Gabriel l'orateur de taverne, etc. 
Comme on le devine aisément, ici comme dans son théâtre, M. Tremblay n'oublie pas ses classiques et nous propose sa propre figure de la Destinée. Faut-il accorder de l'importance aux noms de ses Parques? Violette, ce joli nom de fleur est aussi une couleur mortuaire tout à fait appropriée aux "maladresses" de celle qui le porte. Quant à Rose et Mauve, autres noms de fleurs: elles ne se comportent pas de la même façon que Violette, étant plutôt caractérisées dans l'aspect continu et répétitif de leur "tricotage". Que faire de Florence, la mère omnisciente qui supervise le travail de ses filles? Notons, pour l'instant, qu'elle porte non seulement le nom d'une ville italienne mais que florence est aussi un substantif désignant un tissu ou une fibre soyeuse qu'on file, appelée aussi crin de Florence, qu'on utilise pour tisser ou encore pour les points de suture, en chirurgie... Tout cela ne convient-il pas à la Mère-Destinée, surveillante de tous les fils et tricots de ses filles qui portaient jadis les noms d'Atropos, Clotho et Lachésis? La mère du tricot vital, existentiel, est bien nommée.

On ne sera donc pas étonné que certains lecteurs, fidèles au «prière d'insérer" (ou au texte publicitaire de la couverture arrière) voit dans ce roman un mélange de réalisme et de fantastique. Mais ce serait passer trop vite sur la dimension tragique (même si elle prend des airs bonhommes, ou de "bonnes femmes") qui innerve cette tendre chronique dont nous n'avons ici que le premier volet, puisque l'auteur annonce déjà le deuxième qui s'intitulera Thérèse et Pierrette à l'école des Saints-Anges. Quant au réalisme, il ne va pas jusqu'à une reconstitution documentaire de l'époque : quelques détails importants ou d'ambiance suffisent (le tramway, les spectacles de la Poune, les bonbons à la cenne du «restaurant», etc.). II s'agit bien plutôt d'une évocation de la mentalité populaire des années 1940.

Signalons enfin l'apparition de plusieurs personnages déjà connus dans les pièces de Tremblay: des Belles-Sœurs, on retrouve Germaine Lauzon (la «millionnaire"), Rose Ouimet, Gabrielle Jodoin et Pierrette Guérin. On fait aussi connaissance avec le jeune ménage Marie-Louise et Léopold Brassard qui sont les parents de $\grave{A}$ toi, pour toujours, ta Mari-Lou.

Et la grosse femme enceinte, sur-soulignée par le titre et la couverture? Quel est son rôle? Elle qui n'est jamais désignée autrement (ou alors comme la femme de Gabriel, le fils de Victoire) demeure mystérieuse à bien d'autres titres: notons que cette femme lit Hugo et Balzac; qu'elle est d'origine indienne et qu'elle a droit à une prédiction de Florence: l'enfant qu'elle attend est "multiple» (p. 207)... Elle symbolise déjà toutes les maternités difficiles de l'époque. Elle ne sera sûrement pas à l'image de la Luzina de la Petite Poule d'eau qui, elle aussi, accouche tardivement. 
Ainsi, beaucoup de fenêtres restent à découvrir dans ce récit qui privilégie tellement l'observation secrète, sinon le voyeurisme. Attendons la suite du tricot de Rose, Violette et Mauve : tout bouge déjà dans le ventre des sept femmes enceintes qu'écoute, à la dernière page. Florence, la mère du fil ${ }^{2}$.

Jacques Allard

1. La grosse femme d'à côté est enceinte, de Michel Tremblay, est paru chez Leméac, dans la collection "Roman québécois", en 1978 (323 p.).

2. Je ne résiste pas à une dernière note: à la fin du texte, l'auteur précise que son roman a été écrit de novembre 1977 à août 1978, cette période de neuf mois nous ramenant encore à la gestation fondamentale... 